\title{
Zum Erfolg verurteilt. Die Willensbildung in der großen Koalition seit 2005 am Beispiel der Finanzpolitik
}

\author{
Otto Bernhardt und Anne Deter
}

Die Ende 2005 gebildete große Koalition wird häufig als Zweckbündnis tituliert. „Das ist keine Liebesheirat. Aber insgesamt glaube ich, dass wir eine gute Entscheidung für das Land getroffen haben"1, sagte der seinerzeitige und heutige Vorsitzende der SPD, Franz Müntefering, nach Abschluss der Koalitionsverhandlungen zwischen CDU/CSU und SPD. Koalitionen, ob große ${ }^{2}$ oder kleine ${ }^{3}$, ob in Mehrheitskonstellationen oder in Minderheitsregierungen, stellen selten bis nie eine „Traumhochzeit“ dar, denn die Parteien wollen natürlicherweise lieber allein regieren. ${ }^{4}$ Doch die Zweckbündnisse zur Erreichung gemeinsamer Ziele dürften gerade bei kleinen Koalitionen näher beieinander stehen als bei großen. Schließlich finden sich in einer großen Koalition Parteien zusammen, die eine breite Wählerschicht hinter sich versammeln wollen, und dies oftmals mit abgrenzenden Positionen der einen von der anderen Volkspartei. Sachliche und persönliche Verletzungen im vorangegangenen Wahlkampf können bei so stark in der politischen Meinungsbildung konkurrierenden Parteien tief sein. ${ }^{5}$

Große Koalitionen hat es auf Bundesebene in Deutschland lediglich in den Jahren des Übergangs von einer CDU/CSU-dominierten Bundesregierung hin zu einer Bundesregierung mit SPD-Mehrheit von 1966 bis 1969 und nach dem Ende der rot-grünen Bundesregierung ab 2005 gegeben. Ähnlich verlief die Geschichte bereits in der Weimarer Republik, und so stellt sich auch die Lage auf Ebene der Bundesländer dar. Hier bilden sich häufiger große Koalitionen, bleiben aber in einer Gesamtschau eher die Ausnahme. ${ }^{6}$ Anders als etwa im benachbarten Österreich, wo ÖVP und SPÖ zusammen die am längsten existierende Regierungsform seit Ende des Zweiten Weltkriegs bilden, bevorzugt Deutschland offensichtlich nicht zwei große Fraktionen in der Regierung. Mit dem Ende der DDR hat sich allerdings das Bild großer Koalitionen gewandelt. In einigen Bundesländern ist die Nachfolgepartei der SED, die PDS, die inzwischen nach der Vereinigung mit der WASG den Namen Die Linke trägt, die zweitstärkste Kraft. Diese Entwicklung soll bei der Behandlung großer Koalitionen und deren Willensbildung außer Betracht bleiben, im Fokus stehen vielmehr die bislang allein auf Bundesebene auftretenden großen Koalitionen der Volkspar-

1 Ulrike Brendlin / Julia Topar, Das dramatische Protokoll des Machtwechsels in Berlin, in: BildZeitung vom 11. Oktober 2005.

2 Koalitionen, in denen die beiden stärksten Fraktionen eine Regierung bilden. Da dies mittlerweile in der Bundesrepublik Deutschland keine singuläre Regierungsform mehr darstellt, wird hier „groß“ anstatt „Groß“ geschrieben.

3 Koalitionen, in denen vornehmlich eine stimmenstarke Fraktion mit einer Fraktion mit verhältnismäßig geringerem Stimmenanteil eine Regierung bildet.

4 Vgl. die metaphorischen Vorbetrachtungen bei Sabine Kropp, Regieren in Koalitionen. Handlungsmuster und Entscheidungsbildung in deutschen Länderregierungen, Wiesbaden 2001, S. 13 f.

5 So etwa zur ersten großen Koalition in der Bundesrepublik Deutschland von 1966 bis 1969 Reinhard Schmoeckel/ Bruno Kaiser, Die vergessene Regierung: die große Koalition 1966 bis 1969 und ihre langfristigen Wirkungen, Bonn 1991, S. 79.

6 Vgl. Sabine Kropp, a.a.O. (Fn. 4). 
teien CDU, CSU und SPD, die zusammen mehr als zwei Drittel der Stimmen hinter sich vereinen können.

Im Folgenden soll anhand der zweiten großen Koalition auf Bundesebene am Beispiel der Finanzpolitik erläutert werden, wie Entscheidungen in großen Koalitionen gefunden werden und welchen Einfluss zu welchem Zeitpunkt die im Parlament vertretenen Regierungsfraktionen auf die Gesetzgebung nehmen können. Es soll dargestellt werden, ob sich gerade in großen Koalitionen Rituale und Gremien bilden, die die demokratische Willensbildung in kleinere Zirkel verlagern - nicht immer mit voller Unterstützung der möglicherweise weniger eingebundenen an der Regierung beteiligten Gesamtfraktionen. Oder ist der Einfluss der Regierungsfraktionen in einer großen Koalition nicht größer und agieren diese nicht selbstbewusster? Haben große Koalitionen also besondere Abstimmungsmechanismen entwickelt, weil sie „zum Erfolg verurteilt“" ${ }^{\text {"zind? }}$

Große Koalitionen sind in der Literatur mehrfach beschrieben worden. ${ }^{8}$ Über ihre Willensbildung finden sich aber nur recht wenige Ausführungen, insbesondere zum Kreßbronner Kreis, eine Art Koalitionsausschuss der ersten großen Koalition. ${ }^{9}$ Allerdings fehlen weitgehend Beschreibungen der an der konkreten Willensbildung zu einzelnen Gesetzesvorhaben beteiligten Kreise. Diese Lücke soll hier anhand von Beispielen aus der Gesetzgebung im finanzpolitischen Bereich in der seit 2005 regierenden großen Koalition ansatzweise geschlossen werden.

\section{1. "Übergangsregierung" 1966 bis 1969 und Kreßbronner Kreis}

Nach den Wahlen zum Deutschen Bundestag im Jahre 1961 kam es zu den ersten Koalitionsverhandlungen zwischen CDU/CSU und SPD, angetrieben von Mitgliedern der beiden Fraktionen, die eine große Koalition zur Lösung der nationalen und außenpolitischen Probleme für unerlässlich hielten. Von der Bevölkerung, die von „Traumregierung“ oder „Kabinett der Besten "10 sprach, wurde dieser Wunsch unterstützt. ${ }^{11}$ Aber Bundeskanzler Ludwig Erhard, der 1963 Konrad Adenauer im Kanzleramt gefolgt war, wandte sich entschieden gegen eine große Koalition. Mit den zunehmenden wirtschaftspolitischen Problemen der Bundesrepublik Deutschland und einer Führungsschwäche Erhards sowie einer uneinheitlich agierenden CDU kamen auch in der Bevölkerung Zweifel am politischen System im Westen Deutschlands auf. Mit dem Einzug rechter Parteien in einige Landespar-

7 So der Titel einer Broschüre des Journalisten Franz Schneider, Die große Koalition - zum Erfolg verurteilt?, Mainz 1968.

8 Instruktiv mit weiteren Hinweisen Peter März, Große Koalitionen. Deutsche und weitere Erfahrungen, in: Einsichten und Perspektiven. Bayerische Zeitschrift für Politik und Geschichte, 5. Jg. (2007), Themenheft 1, http://www.km.bayern.de/blz/eup/01_07_themenheft/index.asp (Abruf am 8. März 2009).

9 Weitergehend zur großen Koalition von 1966 bis 1969 Joachim Samuel Eichhorn, „Mehr als einige schöne Trinksprüche“. Die Konsensstrategien der ersten Großen Koalition (1966-1969), in: Zeithistorische Forschungen, 3. Jg. (2006), H. 2, S. 231 - 246.

10 Gerd Buccerius, Ein Kabinett der Besten. Warum Ludwig Erhard den Kanzler Adenauer ablösen muss, in: Die Zeit vom 6. Oktober 1961, S. 1.

11 Rolf Zundel, Die große Koalition - eine demokratische Todsünde?, in: Alois Rummel (Hrsg.), Die Große Koalition 1966-1969. Eine kritische Bestandsaufnahme, Freudenstadt 1969, S. 30. 
lamente sahen sich SPD und CDU gedrängt, ein „zweites Weimar“ abzuwehren und eine Koalition miteinander einzugehen. ${ }^{12}$ Schließlich zerbrach das Bündnis von CDU/CSU und FDP 1966 daran, dass die Union das entstandene Haushaltsdefizit und die immer größer werdende Verschuldung im Haushalt 1967 durch Steuererhöhungen ausgleichen wollte. Die FDP trat aus der Regierung Erhard aus, CDU/CSU nahmen Verhandlungen mit der SPD auf, und am 1. Dezember 1966 wurde die erste große Koalition in der Bundesrepublik geschlossen. ${ }^{13}$

Geführt wurde die Regierung von dem früheren Ministerpräsidenten Baden-Württembergs, Kurt Georg Kiesinger. Dessen Stellvertreter und Außenminister wurde der bisherige Regierende Bürgermeister von West-Berlin und SPD-Vorsitzende Willy Brandt. Die große Koalition sah sich drei großen Aufgaben gegenüber, ohne diese allerdings in einem Koalitionsvertrag zu manifestieren. ${ }^{14}$ Erste wichtige Aufgabe war die Sanierung des maroden Haushalts und die Eindämmung der Staatsschulden sowie die Bekämpfung der ersten Rezession nach 1945. Unter Finanzminister Franz Josef Strauß (CSU) und Wirtschaftsminister Karl Schiller (SPD), die in der Öffentlichkeit den Spitznamen „Plisch und Plum"15 trugen, war es Ziel der großen Koalition, die Wirtschaft recht schnell wieder zu beleben. ${ }^{16}$ Zweitens sollten die noch bestehenden Eingriffsrechte der Alliierten in die Souveränität Deutschlands abgelöst werden. Diese forderten dazu die Verabschiedung der so genannten Notstandsgesetze, „Kainszeichen"17 der großen Koalition, um die Sicherheit ihrer in Deutschland stationierten Truppen gewährleistet zu wissen. Für die Änderung der Verfassung war eine Zweidrittelmehrheit im Bundestag nötig. Besonders daran schieden sich die Geister, da es der Regierung während eines nationalen Notstandes nun möglich sein würde, Grundrechte vorübergehend außer Kraft zu setzen. ${ }^{18}$ Drittens war das Ziel der großen Koalition, das Mehrheitswahlrecht nach britischem oder US-amerikanischem Modell einzuführen, damit nach Wahlen stets eine Partei die absolute Mehrheit erhielte und man nicht mehr auf Koalitionsverhandlungen angewiesen sei. Dieses Vorhaben scheiterte jedoch am Ende an der SPD, nachdem die FDP, um deren parlamentarisches Überleben es dabei ging, sich ihr als potenzieller Koalitionspartner für die nächste Wahlperiode anbot. In diesem Zusammenhang überwarf sich die CDU/CSU mit der FDP und strebte für die kommende

12 Ebenda, S. 31. Instruktiv hierzu Günter Buchstab (Bearb.), Kiesinger: „Wir leben in einer veränderten Welt". Die Protokolle des CDU-Bundesvorstands 1965-1969, Forschungen und Quellen zur Zeitgeschichte, Bd. 50, Düsseldorf 2005, S. XVI - XVII.

13 Vgl. RolfZundel, a.a.O. (Fn. 11), S. 31; Günter Buchstab, a.a.O. (Fn. 12), S. XVIII. Zu den Koalitionsverhandlungen im Einzelnen vgl. Reinhard Schmoeckel / Bruno Kaiser, a.a.O. (Fn. 5), S. 41 -59 .

14 Vielmehr galt die Regierungserklärung vom 13. Dezember 1966 als Vorgabe für das Regierungshandeln, vgl. Joachim Samuel Eichhorn, a.a.O. (Fn. 9), S. 232.

15 Vgl. Reinhard Schmoeckel / Bruno Kaiser, a.a.O. (Fn. 5), S. 65 f. Plisch und Plum ist der Titel einer Bildergeschichte von Wilhelm Busch über zwei ungezogene junge Hunde, die vom alten Kaspar Schlich ertränkt werden sollen. Die beiden ebenso ungezogenen und streitsüchtigen Jungen Paul und Peter retten die Hunde und nehmen sie mit nach Hause. Trotz äußerer Unterschiedlichkeit (der eine gedrungen, der andere hager) begehen die Hunde in völligem Einvernehmen eine Reihe dreister Streiche.

16 RolfZundel, a.a.O. (Fn. 11), S. 32.

17 Ebenda, S. 31.

18 Günter Buchstab, a.a.O. (Fn. 12), S. XXIV - XXVI. 
Bundestagswahl die Alleinregierung mit einer absoluten Mehrheit unter Bundeskanzler Kiesinger an, einem vehementen Befürworter des Mehrheitswahlrechts. ${ }^{19}$

In der großen Koalition von 1966 bis 1969 vermied man es, von Koalitionsausschüs$\operatorname{sen}^{20} \mathrm{zu}$ sprechen, denn die Regierung Kiesinger glaubte, ohne dieses Instrument auszukommen. ${ }^{21}$ Der Kreßbronner Kreis, benannt nach dem Urlaubsort Kiesingers am Bodensee, stellte aber zweifelsohne ein Gremium dar, das die Klärung von Differenzen innerhalb der Regierungskoalition zum Ziel hatte. ${ }^{22}$ Am ersten zwanglosen Treffen mit Kiesinger nahmen nur Willy Brandt (SPD-Chef, Vizekanzler und Außenminister), Herbert Wehner (SPD-Vize und Gesamtdeutscher Minister) und Bruno Heck (CDU-Generalsekretär und Familienminister) teil. Ein Jahr später war der Kreis auf etwa ein Dutzend Teilnehmer gewachsen. Dazu zählten insbesondere Franz Josef Strauß (CSU-Chef und Finanzminister) sowie die Fraktionschefs Helmut Schmidt (SPD) und Rainer Barzel (CDU/CSU). Im Kreßbronner Kreis wurden - meist dienstagmittags einen Tag vor der Kabinettssitzung - Entscheidungen getroffen, bevor sie in offiziellen Gremien wie Kabinett oder Parlament ausführlich diskutiert wurden. ${ }^{23}$ Schmidt und Barzel sorgten dafür, dass die parlamentarischen Hürden reibungslos genommen wurden. ${ }^{24}$ Dazu zählten Gesetze zur Finanzplanung und Steuerpolitik sowie eine Strafrechtsreform. Kiesinger setzte in den Gesprächen auf seine Fähigkeiten als Moderator und nicht auf die Richtlinienkompetenz als Kanzler. Wehner habe sich „am korrektesten " an die Vereinbarungen gehalten, sagte Kiesinger später. ${ }^{25}$ Mit dem Kreßbronner Kreis hatte sich erstmals in der Geschichte der Bundesrepublik Deutschland ein inoffizielles Gremium institutionalisiert, das mehr oder minder konkrete Vorgaben machte. ${ }^{26}$ Wegen seiner kritischen demokratischen Legitimation fand der Kreßbronner Kreis bei Staatsrechtlern und auch bei Journalisten keine oder nur geringe Zustimmung. Schließlich war er

19 RolfZundel, a.a.O. (Fn. 11), S. 35 f.; Günter Buchstab, a.a.O. (Fn. 12), S. XXII - XXIV.

20 Zur Definition und zu den Aufgaben von Koalitionsausschüssen im Allgemeinen Gerhard Moritz Meyer, Die laufende Abstimmung zwischen den Koalitionspartnern. Koalitionsausschüsse und Koalitionsgespräche, in: Friedrich-Naumann-Stiftung (Hrsg.), Probleme von Koalitionsregierungen in Westeuropa, Bonn 1978, S. $101-109$.

21 Ulrich Frank-Planitz, Ein „Koalitionsausschuss“ für die Regierung Kiesinger? Die CDU möchte an den Entscheidungen des Kanzlers stärker beteiligt sein, in: Handelsblatt vom 4. September 1967; Joachim Samuel Eichhorn, a.a.O. (Fn. 9), S. 236.

22 Zum Hintergrund Hermann Otto Bolesch, Seine Wiege stand unter Pflaumenbäumchen und Rosensträuchern, in: Abendzeitung vom 16. Juni 1969. Instruktiv zum Hintergrund und Wirken des Kreßbronner Kreises Joachim Samuel Eichhorn, a.a.O. (Fn. 9), S. 238 f. Neben dem Kreßbronner Kreis gab es noch Ad-hoc-Koalitionsgespräche, vgl. ebenda, S. 242.

23 Vgl. Karl Carstens, Erinnerungen und Erfahrungen, herausgegeben von Kai von Jena und Reinhard Schmoeckel, Boppard am Rhein 1993, S. 357; Reinhard Schmoeckel / Bruno Kaiser, a.a.O. (Fn. 5), S. 75.

24 Vgl. Reinhard Schmoeckel / Bruno Kaiser, a.a.O. (Fn. 5), S. 75; des Weiteren Peter März, a.a.O. (Fn. 8), Kapitel 7, S. 4. Instruktiv zur koordinierenden Aufgabe Kiesingers als Bundeskanzler und zur Bedeutung der Fraktionen Heribert Knorr, Der parlamentarische Entscheidungsprozess während der Großen Koalition von 1966 bis 1969, Meisenheim am Glan, S. 214 - 218.

25 Klaus Bloemer, Wer hat Angst vor F.J.S.?, in: Die Zeit vom 12. April 1974, S. 9, S. 60.

26 Andere halten den Kreßbronner Kreis weder für spezifisch noch für gefährlicher als irgendeinen anderen Koalitionsausschuss, so Rolf Zundel, a.a.O. (Fn. 11), S. 40. Zwar hielten spätere Koalitionsregierungen an der Praxis informeller, regelmäßiger Besprechungen fest, sie stellten aber keine offizielle Institution mehr, vgl. Reinhard Schmoeckel/ Bruno Kaiser, a.a.O. (Fn. 5), S. 76. 
nicht in der Verfassung vorgesehen und stellte eine Art Nebenregierung dar. ${ }^{27}$ Allerdings habe sich der Kreis darauf beschränkt, von Zeit zu Zeit die gesetzgeberischen Zielvorstellungen in einem Katalog festzuhalten und zu überprüfen. ${ }^{28}$

Ein positiver Effekt der großen Koalition war das gestiegene Selbstbewusstsein der Parlamentarier. „Es entsteht ein stärkeres Spannungsverhältnis zwischen dem Parlament als ganzem einerseits und der gesamten Regierung andererseits. (...) Die breite Regierungsmehrheit im Hause wird den einzelnen Fraktionen und einzelnen Abgeordneten mehr Raum als bisher für eigene Initiativen bieten. Vielleicht werden sich auch wechselnde Mehrheiten ergeben, (...) ohne dass dies nun zur Erschütterung (...) [der] Stabilität führen muss“29, prophezeite Helmut Schmidt zu Beginn der Wahlperiode 1966. In der Tat machten es die beiden Koalitionsparteien der Regierung nicht immer leicht und spielten eine besondere Rolle; Gesetze wurden nicht einfach „durchgewunken“, sondern zum Teil heftig diskutiert. ${ }^{30}$ Als bei der Beratung um die Lohnfortzahlung im Bundestag Minister Schiller die Urheberrechte für die Konzertierte Aktion ${ }^{31}$ zur Stabilisierung der Wirtschaft in Anspruch nehmen wollte, wurde er vom eigenen Fraktionsvorsitzenden darauf hingewiesen, dass nur der Bundestag allein und kein außerparlamentarisches Gremium die Gesetze beschließe. ${ }^{32}$ Die Entscheidungen der großen Koalition wurden also nicht durch den Kreßbronner Kreis präjudiziert, sondern es kann als wesentliches Merkmal festgestellt werden, dass es zu einer Versachlichung der Politik mittels informeller Gremien $\mathrm{kam}^{33}$ : „In den zweieinhalb Jahren der Großen Koalition hat sich die politische Landschaft der Bundesrepublik tatsächlich mehr verändert als je zuvor. "34 Kurz vor ihrem Ende fasste Bundeskanzler Kiesinger die Arbeit in der großen Koalition zusammen: „Sie dürfen nicht glauben, dass es leicht gewesen sei, dieses Gespann, das so ganz anders geartet ist als Gespanne von ehedem, mit dem starken Zugpferd neben dem kleinen, übermütigen Pony, wirklich zusammenzuhalten. Das

27 Vgl. ebenda, S. 74. Zur Kritik an Koalitionsausschüssen im Allgemeinen Gerhard Moritz Meyer, a.a.O. (Fn. 20), S. 107 - 109.

28 Vgl. Albert Pfeiffer, Positive Gesamtbilanz trotz Kreßbronner Kreis. Nur die konzertierte Aktion passte nicht in den Rahmen, in: Industrie Kurier Düsseldorf vom 5. Juli 1969.

29 Regierungserklärung vom 13. Dezember 1966, zitiert nach Reinhard Schmoeckel/ Bruno Kaiser, a.a.O. (Fn. 5), S. 78 .

30 Joachim Samuel Eichhorn, a.a.O. (Fn. 9), S. 240.

31 Der Begriff „Konzertierte Aktion“ stammt aus einem Jahresgutachten des Sachverständigenrates zur Begutachtung der gesamtwirtschaftlichen Entwicklung aus dem Jahr 1965, in dem die Einrichtung einer konzertierten Aktion nach ausländischen Vorbildern empfohlen wurde. Der damalige Bundeswirtschaftsminister Karl Schiller (SPD) griff die Empfehlung als ein Mittel zur Steuerung der Konjunktur auf. Unter Schillers Vermittlung traten Vertreter der Regierung, der Gebietskörperschaften, der Gewerkschaften, der Arbeitgeber- und der Unternehmerverbände zusammen. Alle am Wirtschaftsprozess beteiligten Institutionen sollten ihr Verhalten aufeinander abstimmen, um die Wirtschaftskrise jener Jahre zu überwinden. Vgl. Uwe Andersen / Wichard Woyke (Hrsg.), Handwörterbuch des politischen Systems der Bundesrepublik Deutschland, Opladen 2003, S. 607. Zur fraktionellen Abstimmung der Konzertierten Aktion vgl. Klaus Schönhoven, Wendejahre. Die Sozialdemokratie in der Zeit der großen Koalition 1966-1969, Bonn 2004, S. 139 - 141.

32 Albert Pfeiffer, a.a.O. (Fn. 28).

33 Die Versachlichung der Politik drückte sich unter anderem in der Einrichtung von verschiedenen Institutionen wie dem Finanzplanungsrat oder dem Sachverständigenrat aus.

34 Rolf Zundel, a.a.O. (Fn. 11), S. 31.436 Gesetze verabschiedete der Bundestag in der fünften Wahlperiode, Albert Pfeiffer, a.a.O. (Fn. 28). 
war eine der ganz großen Pflichten meiner Regierungstätigkeit, und es wäre deswegen auch Narretei, wenn ich versuchen würde, Zensuren auszuteilen. “35 Die große Koalition 1966 bis 1969 war mäßig populär; Umfragen zufolge waren rund zwei Drittel der Bürger zufrieden. ${ }^{36}$ Nach der Bundestagswahl 1969 bildete Willy Brandt gegen den Willen von Herbert Wehner und Helmut Schmidt, die eine Fortsetzung der großen Koalition vorgezogen hätten, eine Koalition mit der FDP.

\section{Koalitionsverhandlungen und Koalitionsvertrag von 2005}

„Ich halte die so genannte Große Koalition für die beste praktische Möglichkeit, um einigermaßen stabile Regierungsverhältnisse in Deutschland zu schaffen "37, äußerte Gustav Stresemann in einem Telegramm nach Berlin, bevor die zweite große Koalition der Weimarer Republik ihre Arbeit aufnahm. So oder ähnlich haben sich auch die zukünftigen Koalitionspartner nach der Bundestagswahl 2005 geäußert. Anders aber als 1966 musste die große Koalition im Jahre 2005 mehr oder minder improvisiert werden. ${ }^{38}$ In der vorgezogenen Bundestagswahl vom 18. September konnte kein angestrebtes Bündnis, weder eine schwarz-gelbe Koalition aus CDU/CSU und FDP noch eine rot-grüne aus SPD und Bündnis 90/Die Grünen, die absolute Mehrheit der Mandate erreichen. Dies war unter anderem auch dem Einzug der PDS/Die Linke geschuldet, die 8,7 Prozent der Stimmen erhielt. Keine der Parteien im Bundestag war bereit, mit ihr eine Koalition einzugehen. Ob sich dies in Zukunft ändern wird, ist und bleibt Thema heftiger Auseinandersetzungen vornehmlich in der SPD. 2005 war auch die Tolerierung einer rot-grünen Regierung durch Die Linke ausgeschlossen. Die FDP war ihrerseits nicht bereit, einer Koalition zwischen SPD und Grünen beizutreten. ${ }^{39}$ Die am Tag der Wahl ins Gespräch gebrachte Idee eines Bündnisses zwischen CDU/CSU, FDP und den Grünen, nach den Farben der Nationalflagge von Jamaika beziehungsweise Schwampel genannt, scheiterte früh an den zu unterschiedlichen Ausgangssituationen der vier potentiellen Partner. ${ }^{40}$

35 Zitiert nach Dieter Oberndörfer (Hrsg.), Kurt Georg Kiesinger. Die Große Koalition 1966-1969. Reden und Erklärungen des Bundeskanzlers, Stuttgart 1979, S. 295.

36 Nach einer Emnid-Umfrage vom Februar 1969 waren 73 Prozent der CDU/CSU-Anhänger mit der großen Koalition zufrieden, bei der SPD waren es 59 Prozent, bei der FDP 20 Prozent und bei der NPD zwölf Prozent, vgl. Tagesspiegel vom 29. März 1969; RolfZundel, a.a.O. (Fn. 11), S. 37.

37 So Gustav Stresemann, zitiert nach Peter März, a.a.O. (Fn. 8), Kapitel 3, S. 6.

38 Peter März, a.a.O. (Fn. 8), Kapitel 5, S. 5. Zur Ausgangslage der großen Koalition Richard Hilmer / Rita Müller-Hilmer, Die Bundestagswahl vom 18. September 2005: Votum für Wechsel und Kontinuität, in: ZParl, 37. Jg. (2006), H. 1, S. 183 - 218, S. 188 ff. sowie zur Regierungsbildung S. $213 \mathrm{ff}$.

39 „Dass das ganze ,Ampel'-Manöver angesichts der eindeutig ablehnenden Haltung der FDP recht realitätsfern ist, wissen freilich auch die Spitzenleute der Grünen“, schrieb etwa Stephan Löwenstein, Jamaika klingt besser als „Schwampel“, in: FAZ vom 20. September 2005, S. 6.

40 Schwampel steht, angelehnt an die „Ampel“ für die Konstellation SPD-FDP-Grüne, für „Schwarze Ampel“. Claudia Roth, die Parteivorsitzende der Grünen, äußerte sich wie folgt zur Schwampel beziehungsweise Jamaika-Koalition: „Ich war noch nicht in Jamaika, aber ich bin alter ReggaeFan, und das hat herzlich wenig mit der Leitkultur von Herrn Stoiber zu tun." Vgl. ebenda. 
Am 11. November 2005 kam es zwischen CDU/CSU und SPD zur Einigung auf den endgültigen Wortlaut eines Koalitionsvertrages. Nachdem die Parteitage von Union und SPD mit großer Mehrheit dem Vertragswerk zugestimmt hatten, wurde der Koalitionsvertrag am 18. November von den Vorsitzenden der drei Parteien unterzeichnet. Am 22. November 2005 wurde Angela Merkel zur Bundeskanzlerin gewählt und die Minister ihres Kabinetts ernannt. ${ }^{41}$ Der Koalitionsvertrag betont fünf besonders reformbedürftige Politikfelder. Gemeinsamkeiten zwischen den Koalitionspartnern erscheinen hierbei je geringer desto konkreter die Umsetzungsvorstellungen werden (siehe Tabelle 1).

\begin{tabular}{|c|c|c|c|}
\hline $\begin{array}{c}\text { Reform- } \\
\text { projekt }\end{array}$ & Reformziel & $\begin{array}{c}\text { Parteipositionen } \\
\text { (CDU/CSU - SPD) }\end{array}$ & $\begin{array}{l}\text { Programmatische } \\
\text { Schnittmenge }\end{array}$ \\
\hline $\begin{array}{l}\text { Familien- } \\
\text { politik }\end{array}$ & $\begin{array}{l}\text { Familienfreundliche } \\
\text { Gesellschaft, Reaktion auf } \\
\text { demographischen Wandel, } \\
\text { Kompatibilität von Familie } \\
\text { und Beruf }\end{array}$ & $\begin{array}{l}\text { Kindergeld vs. öffentlich } \\
\text { ausgebaute Kinderversorgung; } \\
\text { Ehegattensplitting vs. Splitting } \\
\text { nur bei Ehen mit Kindern }\end{array}$ & $\begin{array}{l}\text { Relativ groß; } \\
\text { Kompromisse } \\
\text { möglich }\end{array}$ \\
\hline Föderalismus & $\begin{array}{l}\text { Politikentflechtung, klarere } \\
\text { Kompetenzteilung zwischen } \\
\text { Bund und Ländern } \\
\end{array}$ & $\begin{array}{l}\text { Stärkung von Ländern vs. } \\
\text { Gesamtverpflichtung des } \\
\text { Bundes }\end{array}$ & $\begin{array}{l}\text { Mittelgroß; } \\
\text { Kompromisse } \\
\text { prinzipiell möglich } \\
\end{array}$ \\
\hline Gesundheit & $\begin{array}{l}\text { Senken der Lohnnebenkosten, } \\
\text { langfristige Finanzierbarkeit, } \\
\text { ausreichende Gesundheitsleis- } \\
\text { tungen, Verteilungsgerechtig- } \\
\text { keit }\end{array}$ & $\begin{array}{l}\text { Bürgerversicherung vs. } \\
\text { Kopfpauschale; Beitrags- vs. } \\
\text { stärkere Steuerfinanzierung; } \\
\text { Verminderung der Versor- } \\
\text { gungsleistung vs. Ausdehnung } \\
\text { der Finanzierungsbasis }\end{array}$ & $\begin{array}{l}\text { Klein; Kompromisse } \\
\text { prinzipiell möglich }\end{array}$ \\
\hline $\begin{array}{l}\text { Staatsver- } \\
\text { schuldung }\end{array}$ & $\begin{array}{l}\text { Entschuldung durch } \\
\text { Kosteneinsparungen, } \\
\text { Bürokratie- und Subventions- } \\
\text { abbau, Ausgabenkürzung, } \\
\text { Steuererhöhungen }\end{array}$ & $\begin{array}{l}\text { Ausgaben kürzen vs. } \\
\text { Einnahmen erhöhen }\end{array}$ & $\begin{array}{l}\text { Mittelgroß; } \\
\text { Kompromisse } \\
\text { möglich }\end{array}$ \\
\hline Arbeitsmarkt & $\begin{array}{l}\text { Senkung von Lohnzusatz- } \\
\text { kosten und Jugendarbeits- } \\
\text { losigkeit, aktivierende } \\
\text { Arbeitsmarktpolitik }\end{array}$ & $\begin{array}{l}\text { Weitere Deregulierung vs. } \\
\text { staatlich gestützte Aktivierung; } \\
\text { Angebotsseite entlasten vs. } \\
\text { Binnennachfrage stärken }\end{array}$ & $\begin{array}{l}\text { Klein; Kompromisse } \\
\text { eher unwahrschein- } \\
\text { lich }\end{array}$ \\
\hline \multicolumn{4}{|c|}{$\begin{array}{l}\text { Quelle: Wolfgang Merkel, Durchregieren? Reformblockaden und Reformchancen in Deutschland, in } \\
\text { Jürgen Kocka, WZB-Jahrbuch 2006. Zukunftsfähigkeit Deutschlands: Sozialwissenschaftliche Essays, } \\
\text { Berlin 2007, S. } 27 \text { - 45, S. 38, mit eigenen Ergänzungen. }\end{array}$} \\
\hline
\end{tabular}

Der Koalitionsvertrag enthält zu den eher als technisch zu bezeichnenden Fragen der Arbeitsweise und damit der Willensbildung in der großen Koalition lediglich zwei Seiten. Wörtlich heißt es darin: „Die Koalitionspartner CDU, CSU und SPD werden ihre Arbeit in Parlament und Regierung laufend und umfassend miteinander abstimmen und zu Verfahrens-, Sach- und Personalfragen Konsens herstellen." Nach Einschätzung einiger Beobachter ist das Bundeskabinett als Grundlage und Folge dieser Abstimmungsvorgaben in der

41 Zur Regierungsbildung im Einzelnen mit zahlreichen weiterführenden Hinweisen Uwe Thaysen, Regierungsbildung in der Bundesrepublik Deutschland: Daten zum Start der Regierung Merkel 2005/2006, in: ZParl, 35. Jg. (2006), H. 3, S. $582-610$. 
großen Koalition seit 2005 ,zunehmend zum Ort kollektiver Willensbildung geworden, keineswegs mehr Notariat der Bundesregierung “42. So finden jeden Mittwoch vor den Sitzungen des Bundeskabinetts jeweils gegen 8:15 Uhr getrennt nach CDU/CSU- und SPDZugehörigkeit Beratungen der Minister statt, an denen maßgebliche Vertreter der Fraktionsführungen teilnehmen. Danach kommt es um 9:30 Uhr zu einer vertraulichen Abstimmung zwischen der Bundeskanzlerin und ihrem Stellvertreter. ${ }^{43}$

Die Bundesregierung achtet bei der Gesetzgebung nicht nur darauf, dass die Vorlagen nach Kabinettsbeschluss auch den Bundestag passieren können, wobei stets das so genannte „Strucksche Gesetz“ 44 gilt: „Kein Gesetz verlässt den Bundestag so, wie es den Bundestag erreicht hat." Vielmehr werden Entwürfe oft schon in ihrer Entstehungsphase mit den Regierungsfraktionen abgestimmt. Dies steht zum einen im Einklang mit den Strukturmerkmalen des „neuen Dualismus“, der für parlamentarische Regierungssysteme typischen Form der Gewaltenteilung. Zum anderen ist es funktional notwendig, denn bei einer Abstimmung nur zwischen federführendem und mitberatenden Ministerien würden die unterschiedlichen Interessen der Regierungsfraktionen nicht genügend Berücksichtigung finden. Die Vorstellungen der Bundestagsfraktionen der Regierungskoalition fließen entweder informell über Kontakte auf der Arbeitsebene oder durch die Einsetzung spezieller Arbeitsgruppen, beispielsweise bei der Unternehmenssteuer- oder der Erbschaftsteuerreform, bereits vor Kabinettsbeschluss in die Gesetzesvorhaben ein. Formalere Verfahren wie die Bestimmung von Arbeitsgruppen zur Vorbereitung von wichtigen Gesetzesvorhaben und Vorgaben, ob und wie ein bedeutendes Gesetz erstellt wird als auch die Auflösung wesentlicher einzelner Streitpunkte zwischen den Koalitionsfraktionen, werden im Koalitionsausschuss geklärt.

\section{Innerfraktionelle Willensbildung zur Vorbereitung finanzpolitischer Entscheidungen}

Bereits in der großen Koalition 1966 bis 1969 gab es wiederholt den Vorwurf, „die eigene Mannschaft erledige unpopuläre Arbeiten im Maschinenraum, die Mannschaft des Gegenspielers lasse sich auf Deck besonnen “45. In Anbetracht der gegenwärtigen Mehrheitsverhältnisse könnte man annehmen, dass die Fraktionsmitglieder die Regierung vor sich „hertreiben“. ${ }^{46}$ Abgeordneten, die eine andere Ansicht vertreten als ihre Gesamtfraktion, könnte in einer großen Koalition breiterer Raum gegeben werden. Anders ist dies bei knappen Mehrheiten, die Fraktionsdisziplin unabdingbar machen. ${ }^{47}$ Trotzdem prägt in der 16. Wahlperiode ein weitgehend geschlossenes Auftreten das Bild der beiden Regierungsfraktionen. Deren Vorsitzende, Volker Kauder und Peter Struck, füllen ihre Rolle anders aus als

42 Karl-Rudolf Korte, Koalition der Willigen. Warum die Bundesregierung ihr Potenzial nicht ausschöpft, in: IP, 13. Jg. (2007), H. 2, S. 90 - 91, S. 90.

43 Vgl. Peter März, a.a.O. (Fn. 8), Kapitel 11, S. 3 f.

44 Tina Hildebrandt / Elisabeth Niejahr, Das ABC der Politik. Verstehen Sie Ihre Regierung noch? Was Politiker sagen, ist selten das, was sie meinen, in: Die Zeit vom 11. Oktober 2007, S. 12.

45 Peter März, a.a.O. (Fn. 8), Kapitel 7, S. 4.

46 Karl-Rudolf Korte, a.a.O. (Fn. 42), S. 91.

47 Vgl. den Artikel über Mitglieder des Bundestags, die sich der Fraktionsdisziplin nicht unterwerfen, Dirk Kurbjuweit, Die Unbeugsamen, in: Der Spiegel vom 22. März 2008, S. 60 - 64. 
Rainer Barzel und Helmut Schmidt: „Zum einen sind beide keineswegs jene dynamischen Nachwuchskräfte wie ehedem, die sich erkennbar profilieren, um Optionen für spätere Spitzenfunktionen zu gewinnen. Beide zeichnen sich auch nicht dadurch aus, dass sie sich besonders forciert bestimmter Materien annehmen, um so Eigenprofil zu gewinnen." 48 Aber nicht allein die Fraktionsvorsitzenden bestimmen die Richtung im Bundestag. Am Beispiel des Bereichs Finanzen, in dem neben dem Bereich Recht die meisten Gesetze verabschiedet werden, zeigt sich ein erheblicher Zeitaufwand allein für die fraktionsinterne Meinungsbildung und Abstimmung. So wendet etwa der Sprecher der Arbeitsgruppe (AG) Finanzen der Unionsfraktion pro Sitzungswoche durchschnittlich etwa ein Dutzend Stunden hierfür auf. So oder ähnlich gestaltet sich die Meinungsbildung in allen Politikfeldern.

Der geschäftsführende Vorstand ${ }^{49}$ der CDU/CSU-Bundestagsfraktion tagt jeweils am Montag einer Sitzungswoche um 15:00 Uhr und der Gesamtfraktionsvorstand ${ }^{50}$, dem die Sprecher aller Arbeitsgruppen kraft Amtes angehören, anschließend um 16:00 Uhr. Wichtige finanzpolitische Themen werden dort entweder vom zuständigen Stellvertretenden Fraktionsvorsitzenden oder vom Sprecher vorgetragen. Die Entscheidung, wer vorträgt, liegt beim Ersten Parlamentarischen Geschäftsführer in Absprache mit dem zuständigen Stellvertretenden Fraktionsvorsitzenden.

In jeder Sitzungswoche lädt der Stellvertretende Vorsitzende der Unionsfraktion Michael Meister Abgeordnete aus den Arbeitsbereichen, für die er zuständig ist - Haushalt, Finanzen und Wirtschaft - zu einer knapp anderthalbstündigen Besprechung ein. Teilnehmer sind der wirtschaftspolitische Sprecher Laurenz Meyer, der haushaltspolitische Sprecher Steffen Kampeter und der finanzpolitische Sprecher Otto Bernhardt. Der Vorsitzende des Parlamentskreises Mittelstand (PKM), Michael Fuchs, der erste stellvertretende Vorsitzende des PKM, Ernst Hinsken, der Sprecher des Arbeitskreises Haushalt und Finanzen der CSULandesgruppe, Bartholomäus Kalb, und die stellvertretenden Arbeitsgruppensprecher, die in der CDU/CSU-Bundestagsfraktion Obleute genannt werden, Leo Dautzenberg, Norbert Barthle und Joachim Pfeiffer sowie der Vorsitzende des Finanzausschusses, Eduard Oswald, komplettieren diese Zusammenkunft. Außerdem sind die beiden Referenten des Stellvertretenden Vorsitzenden anwesend. In diesen Sitzungen geht es um eine allgemeine Abstimmung wichtiger Fragen der Haushalts-, Finanz- und Wirtschaftspolitik.

Die AG Finanzen tagt ähnlich wie die weiteren Arbeitsgruppen aller Fraktionen normalerweise in jeder Sitzungswoche am Dienstagvormittag von 09:00 Uhr bis 10:30 Uhr intern, das heißt, neben den 13 CDU/CSU-Mitgliedern im Finanzausschuss haben lediglich die Referenten der AG und die Abgeordneten Zutritt. Zusätzlich wird das Bundeskanzleramt durch den zuständigen Gruppenleiter und die Referatsleiter der Bereiche Steuern und Finanzen vertreten. Auf der Tagesordnung stehen grundsätzlich alle finanzpolitischen Fragen. In der Regel wird diese Sitzung um 10:30 Uhr um die zuständigen Referenten der so genannten B-Länder, das heißt der Länder, bei denen der Regierungschef der Union

48 Peter März, a.a.O. (Fn. 8), Kapitel 14, S. 2.

49 Zur Zusammensetzung http://www.cducsu.de/Titel_Fraktion/TabID_19/SubTabID_20/Vorstand.aspx (Abruf am 8. März 2009).

50 Der Fraktionsvorstand besteht aus dem Geschäftsführenden Vorstand, den Vorsitzenden der Arbeitsgruppen (Sprechern), den Vorsitzenden der soziologischen Gruppen und 15 weiteren Mitgliedern (Beisitzern). Die Fraktion und ihr Aufbau werden beschrieben unter http://www.cducsu. de/Titel_Organisation/TabID_19/SubTabID_62/Texte.aspx (Abruf am 8. März 2009). 
angehört, erweitert. ${ }^{51}$ Auf der Tagesordnung stehen durchgängig folgende Punkte: Vorbereitung der Finanzausschuss-Sitzung, Initiativen, Plenum der Woche sowie aktuelle finanzund steuerpolitische Fragestellungen.

Jeweils am Dienstag einer Sitzungswoche um 12:45 Uhr lädt der Fraktionsvorsitzende die Mitglieder des geschäftsführenden Fraktionsvorstandes und die 21 Sprecher der Arbeitsgruppen ein, um Fragen, die in den Arbeitsgruppen offengeblieben sind, noch vor der Fraktionssitzung abzuklären. Bei diesem Treffen handelt es sich streng genommen um kein Entscheidungsgremium, dennoch spielt diese Runde eine nicht unerhebliche Rolle bei der Meinungsbildung. Von den Mitarbeitern nehmen nur Referenten des Fraktionsvorsitzenden und seines ersten Stellvertreters an diesen Sitzungen teil.

Dienstags in einer Sitzungswoche findet um 15:00 Uhr eine Fraktionssitzung der CDU/ CSU statt - ähnlich wie bei den anderen im Bundestag vertretenen Fraktionen. Sie beginnt mit einem kurzen Bericht des Fraktionsvorsitzenden, an den sich eine allgemeine Aussprache anschließt. Die Bundeskanzlerin nimmt in der Regel an den Fraktionssitzungen teil und gibt im Anschluss an den Fraktionsvorsitzenden einen kurzen Bericht. Sodann werden normalerweise zwischen drei und sechs wichtige politische Fragen behandelt, zu denen einleitend entweder der zuständige Stellvertretende Fraktionsvorsitzende oder der zuständige Sprecher Stellung bezieht. Häufig finden später Abstimmungen statt. Lediglich in personellen Fragen wird im Einzelfall geheim abgestimmt, in Sachfragen grundsätzlich nicht.

Für jedes Gesetzesvorhaben gibt es eine federführende und zumeist mehrere mitberatende Arbeitsgruppen. Bei wichtigen Vorhaben ist es erforderlich, dass der Berichterstatter der federführenden AG in Kontakt mit den Berichterstattern der mitberatenden Arbeitsgruppen die nötige Koordination und Abstimmung sicherstellt. Es kommt hierbei allerdings auch vor, dass der Sprecher des federführenden Bundestagsausschusses zu einer arbeitsgruppenübergreifenden Sitzung einlädt. Dies war zum Beispiel im Zusammenhang mit der Besteuerung von Biodiesel mehrere Male erforderlich. Eingeladen wurden die zuständigen Abgeordneten mit ihren entsprechenden Referenten beziehungsweise Mitarbeitern, zum Beispiel aus den Bereichen Wirtschaft, Haushalt, Umwelt, Landwirtschaft und Energie.

Der Abstimmung mit den Bundesländern dient insbesondere die Einladung der zuständigen Referenten aus den B-Ländern in die erweiterten Arbeitsgruppensitzungen der CDU/ CSU. In der Praxis führt dies regelmäßig nur dazu, dass die B-Referenten Informationen aufnehmen und in die Landesministerien weitergeben. Ein wichtiges Koordinationsinstrument ist die Teilnahme bestimmter Abgeordneter an den Abstimmungsgesprächen des Bundesrates. Im Bereich Finanzen werden etwa zu den vorbereitenden Sitzungen für den Finanzausschuss des Bundesrates, der etwa jeden dritten Donnerstag stattfindet, sowohl der zuständige Stellvertretende Fraktionsvorsitzende als auch der Sprecher und der Vorsitzende der entsprechenden AG der CSU und die Referenten der genannten drei Abgeordneten eingeladen. Hier findet oft ein reger Gedankenaustausch statt.

51 Mit Ausnahme der Arbeitsgruppe Haushalt, bei der die Mitarbeiter der B-Bundesländer überhaupt keinen Zutritt zu der Arbeitsgruppensitzung haben, sind die Ländervertreter in allen anderen Arbeitsgruppen von Beginn der Sitzungen an anwesend. Die Arbeitsgruppe Finanzen hat sich für eine Zweiteilung der Sitzungen (ohne und mit Vertreter der B-Bundesländer) entschieden, um zunächst vertraulich in einem internen Kreis aktuelle politische Fragen zu diskutieren. Die Vertreter der Länder werden anschließend umfangreich unter anderem durch die Fraktionsreferenten informiert - allerdings ist nur ein kleiner Teil der Länderreferenten tatsächlich anschließend an Auskünften interessiert. 
In der Regel bitten mehrere Dutzend Verbände, zum Teil auch Einzelpersonen und Einzelfirmen, sowohl die Berichterstatter als auch die Sprecher, die zuständigen Stellvertretenden Fraktionsvorsitzenden, teilweise auch die Fraktionsvorsitzenden um interne Gespräche. Während letztere schon aus Zeitgründen nur selten darauf eingehen können, ist die Gesprächsbereitschaft der Stellvertretenden Fraktionsvorsitzenden schon deutlich größer, und die Sprecher versuchen, allen Gesprächswünschen entweder selber oder durch den zuständigen Berichterstatter zu entsprechen. Häufig werden solche Termine von mehreren Abgeordneten gemeinsam wahrgenommen, und oft nimmt zusätzlich der zuständige Referent der Arbeitsgruppe daran teil, schon aus Koordinationsgründen. Im Zusammenhang mit wichtigen Gesetzesvorhaben, wie zum Beispiel der Unternehmenssteuer- und der Erbschaftsteuerreform, hat allein der Sprecher der AG Finanzen der CDU/CSU-Fraktion mehrere Dutzend Verbandsgespräche geführt.

\section{Interfraktionelle Willensbildung zur Finanzpolitik}

Die gemeinsamen Berichterstattergespräche von Union und SPD sind ein wichtiges Entscheidungsgremium, das die Grundlage der Zusammenarbeit der beiden Fraktionen der großen Koalition bildet. An diesen Sitzungen nehmen neben den beiden Berichterstattern (im Einzelfall auch mehrere Berichterstatter von beiden Regierungskoalitionen) die zuständigen Referenten der AG und in Abstimmung Vertreter des zuständigen Ministeriums teil. Wenn sich die Berichterstatter von Union und SPD nicht einigen können, werden entweder die Fraktionssprecher hinzugezogen oder das Thema wird auf die Tagesordnung der Koordinierungsrunde der Sprecher, der so genannten Spiller-Bernhardt-Runde gesetzt. Wenn dort keine Einigung erzielt wird, müssen die beiden zuständigen Stellvertretenden Fraktionsvorsitzenden Joachim Poss und Michael Meister unter Teilnahme der finanzpolitischen Sprecher den nächsten Koordinierungsversuch unternehmen. Wenn es auch dort zu keiner Übereinkunft kommt, befassen sich die Fraktionsvorsitzenden, die Struck-KauderRunde, die zumeist am Dienstagmorgen einer Sitzungswoche zusammenkommt, mit der Fragestellung. Scheitert auch diese, sind Kanzlerin und Vizekanzler gefragt, jetzt also die Merkel-Steinmeier-Runde, und danach wird der Koalitionsausschuss mit der Problematik befasst. Wenn Einigkeit in allen strittigen Punkten zwischen den Koalitionsfraktionen erzielt worden ist, findet normalerweise eine Sitzung der Berichterstatter sämtlicher Fraktionen des Bundestages statt, an der außerdem die zuständigen Referenten der Arbeitsgruppen und Vertreter des Bundesministeriums der Finanzen teilnehmen. In jeder Sitzungswoche finden allein für den Bereich Finanzen normalerweise mehrere solcher Berichterstattergespräche statt.

Die Sprecher der einzelnen Arbeitsgruppen bilden die Grundlage der parlamentarischen Zusammenarbeit zwischen den Koalitionspartnern. ${ }^{52}$ Die Runde der finanzpolitischen Sprecher, Otto Bernhardt und Hans-Ulrich Krüger, vormals Jörg-Otto Spiller ${ }^{53}$, trifft sich am

52 Zur Rolle der Arbeitsgruppenvorsitzenden insgesamt Anne Sophie Petersen / Viktoria Kaina, „Die Fäden habe ich in der Hand“: Arbeitsgruppenvorsitzende der SPD- und CDU/CSU-Bundestagsfraktionen, in: ZParl, 36. Jg. (2007), H. 2, S. $243-260$.

53 Bei der SPD Bundestagsfraktion hat es während der 16. Wahlperiode einen Wechsel in der Sprecherfunktion für die Arbeitsgruppe Finanzen vom Berliner Abgeordneten Jörg-Otto Spiller zu 
Montag einer jeden Sitzungswoche von 12:15 Uhr bis 13:15 Uhr. Eingeladen wird abwechselnd vom SPD- beziehungsweise CDU/CSU-Sprecher. Die Tagesordnung wird im Vorwege von den Referenten der Arbeitsgruppen abgestimmt und vorbereitet. Zur Klärung im Vorfeld gehört auch die Frage, wer vom Finanzministerium an dieser Sitzung teilnimmt. In der Regel ist es die zuständige Parlamentarische Staatsekretärin ${ }^{54}$, darüber hinaus im Einzelfall der oder die zuständigen Abteilungsleiter und gegebenenfalls die mit den Sachfragen betrauten Referenten. Von Seiten der Fraktionen sind neben den Sprechern und deren Referenten regelmäßig der Vorsitzende des Finanzausschusses, Eduard Oswald, und der Vorsitzende des Arbeitskreises Haushalt und Finanzen der CSU-Landesgruppe (das heißt in den ersten zwei Jahren Georg Fahrenschon und anschließend Bartholomäus Kalb) ${ }^{55}$ sowie der Referent dieses Arbeitskreises anwesend. Hinzu kommen die Obleute, das heißt die stellvertretenden Arbeitsgruppensprecher und im Einzelfall die zuständigen Berichterstatter. Im Mittelpunkt stehen die Vorbereitung der Sitzungswoche, die Abstimmung, wie sich die Koalitionsfraktionen zu Fragestellungen einlassen sowie Themen, die auf der Berichterstatterebene nicht einvernehmlich gelöst werden konnten.

Beim Gespräch der Stellvertretenden Fraktionsvorsitzenden entsprechen Leitung und Vorbereitung der Runde der finanzpolitischen Sprecher. Michael Meister ist der zuständige Stellvertretende Fraktionsvorsitzende für die Bereiche Finanzen, Haushalt und Wirtschaft bei der Union und Joachim Poßsein Gegenstück für Finanzen und Haushalt bei der SPD. Diese Runde tagt in der Regel ebenfalls am Montag einer jeden Sitzungswoche von 13:15 Uhr bis 14:15 Uhr. Teilnehmer sind neben den beiden Stellvertretenden Fraktionsvorsitzenden deren Referenten sowie die finanzpolitischen und haushaltspolitischen Sprecher der großen Koalition. Das Bundesministerium der Finanzen ist durch die zuständige Staatsekretärin und gegebenenfalls durch weitere Personen vertreten. Im Mittelpunkt der Sitzung stehen gewöhnlich Themen, die auf der Ebene der Sprecher nicht zufriedenstellend behandelt werden konnten. Während in den ersten zwei Jahren der 16. Wahlperiode die Runde fast in jeder Sitzungswoche einberufen wurde, hat sich der Rhythmus der Sitzungen deutlich verringert. Viele Probleme werden inzwischen von den Berichterstattern und den Sprechern der Arbeitsgruppen gelöst.

Ein Großteil der parlamentarischen Arbeit spielt sich in den Ausschüssen ab, die auf Beschluss des Bundestages für die Dauer der gesamten Wahlperiode gebildet werden. Der Arbeitsbereich des Finanzausschusses deckt sich im Wesentlichen mit den Zuständigkeiten des Bundesministeriums der Finanzen (mit Ausnahme der Haushaltspolitik). Aufgabe des Ausschusses ist es, Gesetzentwürfe, Anträge, Berichte, Entschließungen sowie Vorlagen der Europäischen Union insbesondere aus dem Bereich der Steuerpolitik federführend zu beraten. Die Abgeordneten diskutieren im Ausschuss zudem Vorlagen aus den Bereichen Geld, Kredit-, Finanz- und Kapitalmarkt sowie Versicherungen. Bei komplizierten Vorhaben lädt der Finanzausschuss häufig Fachleute zu einem internen Gespräch ein, um unterschiedliche

Hans-Ulrich Krüger gegeben, Schiller übergab aus Altersgründen sein Amt, vgl. FAZ vom 13. März 2008, S. 19.

54 Vormals MdB Barbara Hendricks, die inzwischen Schatzmeisterin der SPD ist; seit November 2007 ist MdB Nicolette Kressel die zuständige Parlamentarische Staatssekretärin im Bundesfinanzministerium.

55 Kalb trat Ende November 2007 die Nachfolge von Georg Fahrenschon an, der als Staatssekretär ins Bayerische Finanzministerium wechselte, vgl. Passauer Neue Presse vom 29. November 2007. 
Argumente zu hören und den eigenen Entscheidungsprozess abzusichern. Zu praktisch allen wichtigen Gesetzen werden öffentliche Sachverständigenanhörungen durchgeführt, über die ein Wortprotokoll angefertigt wird und die häufig, zumindest ausschnittsweise, auch vom Fernsehsender Phönix übertragen werden. Bis zu 80 Fachleute aus Verbänden und Wissenschaft werden zu solchen Sitzungen eingeladen, die in der Regel mehrere Stunden dauern.

In der großen Koalition ist die Bedeutung des Koalitionsausschusses gestiegen. Während die rot-grüne Bundesregierung dieses Gremium zunächst nur als Instrument zur Schlichtung von Konflikten ansah, das nicht regelmäßig zu tagen brauchte, institutionalisierte die große Koalition es seit 2005 und begrenzt es nicht auf eine „Feuerwehrfunktion“. Im Koalitionsvertrag heißt es dazu im Kapitel „Arbeitsweise der Koalition“: „Die Koalitionspartner treffen sich regelmäßig mindestens einmal monatlich zu Koalitionsgesprächen im Koalitionsausschuss. Darüber hinaus tritt er auf Wunsch eines Koalitionspartners zusammen. Er berät Angelegenheiten von grundsätzlicher Bedeutung, die zwischen den Koalitionspartnern abgestimmt werden müssen, und führt in Konfliktfällen Konsens herbei. Ihm gehören Kanzler, Vizekanzler, Fraktionsvorsitzende (bei der CDU/CSU-Fraktion auch der erste stellvertretende Fraktionsvorsitzende) und, soweit darunter nicht die Parteivorsitzenden sind, die Parteivorsitzenden an." Die so genannte „Siebenerrunde“ besteht aus der Bundeskanzlerin, ihrem Stellvertreter sowie Fraktions- und Parteivorsitzenden ebenso wie den Generalsekretären der Parteien. Je nach Bedarf finden auch erweiterte Koalitionsausschüsse statt, so etwa der Zwanziger-Ausschuss bei der Gesundheitsreform. Anders als vorgesehen hat der Koalitionsausschuss bislang weniger als monatlich getagt. ${ }^{56}$ Die Kanzlerin gilt hierbei ebenso wie im Kabinett als Koordinatorin. ${ }^{57}$ Neben dem Koalitionsausschuss existiert mittlerweile auch eine „Dreierrunde“ der Parteivorsitzenden.

Die große Koalition hat also insgesamt mit einem Zuwachs an informellen Gremien umzugehen, die den normalen Koordinationsbedarf gegenüber den Parteien, Fraktionen und den Ressorts erhöhen.

\section{Drei beispielhafte Gesetzgebungsverfahren aus der Finanzpolitik}

Sofern es sich um vorwiegend fachliche Fragestellungen handelt, bleiben die Entscheidungen fast vollständig auf der Ebene der zuständigen Berichterstatter, etwa bei der 9. Novelle des Versicherungsaufsichtsgesetzes. Die Arbeitsgruppenvorsitzenden sowie die Stellvertretenden Fraktionsvorsitzenden werden allein bei Fragen eingeschaltet, die auf der jeweils unteren Ebene nicht geklärt werden können. Beispielhaft mag hier das Jahressteuergesetz 2008 sein. Die große Koalition prägende Vorhaben wie die Unternehmenssteuerreform werden bereits von Gremien vorbereitet, die sich aus Vertretern der Bundesregierung, der Regierungsfraktionen und der Bundesländer zusammensetzen. Während bezogen auf den Kapitalmarkt zumeist Einvernehmen zwischen den Berichterstattern der Koalitionspartner hergestellt werden kann, werden zumeist einige Punkte der Steuergesetzgebung mindestens auf die Ebene der finanzpolitischen Sprecher oder der Stellvertretenden Fraktionsvorsitzenden gegeben.

$56 \mathrm{Zu}$ den einzelnen Sitzungen des Koalitionsausschusses von 2005 bis 2007 Wolfgang Rudzio, Informelles Regieren. Koalitionsmanagement der Regierung Merkel, in: APuZ, B 16 (2008), S. 11 $-16$.

57 Vgl. Karlheinz Niclauß, Kiesinger und Merkel in der Großen Koalition, in: Das Parlament vom 14. April 2008, S. 6. 
Die Unterschiede zwischen den Koalitionspartnern SPD und CDU/CSU sind hier häufig schon in der Grundeinstellung zu deutlich. Vereinfacht ausgedrückt begehren die Sozialdemokraten eher eine höhere Steuerlast für Einkommensstärkere und eine umfassende Mitwirkung des Staates an Entscheidungen, ausgedrückt in einer hohen Staatsquote, während die Union sich jeweils möglichst niedrige Werte zum Ziel gesetzt hat. Diese Differenzen finden sich in den zum Teil langwierigen Prozessen der Gesetzgebung wieder.

\subsection{Versicherungsaufsichtsgesetz-Novelle}

Ein Beispiel für ein Gesetzgebungsverfahren, bei dem alle wesentlichen Entscheidungen auf Ebene der Berichterstatter abgesprochen wurden, ist die 9. Novelle des Versicherungsaufsichtsgesetzes (VAG). ${ }^{58}$ Anlass für die Überarbeitung war ein Urteil des Bundesverfassungsgerichts (BVerfG) aus dem Jahr 2005, mit dem das VAG für verfassungswidrig erklärt wurde. Das BVerfG hatte unter anderem die Regelungen über die aufsichtsrechtliche Genehmigung der Übertragung von bestehenden Lebensversicherungsverträgen eines Versicherungsunternehmens auf ein anderes beanstandet, weil nicht ausreichend sichergestellt sei, dass bei der Genehmigung die Belange des Versicherten gewahrt werden. Es hatte dem Gesetzgeber daher aufgegeben, bis zum 31. Dezember 2007 eine den verfassungsrechtlichen Anforderungen entsprechende Regelung zu treffen. Daneben bestand Bedarf für die Anpassung der Versicherungsaufsicht an Veränderungen internationaler Standards für die Finanzaufsicht, insbesondere hinsichtlich des internen Risikomanagements der Unternehmen. Außerdem wurde das Verfahren der Mindestüberschussbeteiligung der Versicherten in der Lebensversicherung vereinfacht. ${ }^{59}$ Politisch strittig war bei diesen eher technischen Fragestellungen lediglich, ob Erleichterungen für Pensionsfonds ${ }^{60}$ eingeführt werden sollten. Alle sechs bedeutenden Punkte, die sich aus Gesprächen mit Vertretern verschiedener Interessengruppen und aus der Anhörung zum Gesetzgebungsverfahren selbst ergaben, konnten einvernehmlich auf der Ebene der Berichterstatter gelöst werden. Vor Verabschiedung des Gesetzentwurfs im Finanzausschuss luden die Berichterstatter der großen Koalition die zuständigen Abgeordneten der Oppositionsfraktionen zu einem gemeinsamen Berichterstattergespräch ein, um die beabsichtigten Änderungen zu besprechen. Schließlich wurde das Gesetz mit den Stimmen der Fraktionen der CDU/CSU, SPD und FDP gegen die Stimmen der Grünen bei Stimmenthaltung der Linken verabschiedet.

\subsection{Jahressteuergesetz 2008}

Beim Jahressteuergesetz 2008 wurden zwar die meisten wichtigen Entscheidungen auf Ebene der Berichterstatter besprochen und geklärt, einige schwierige Fragen verblieben aber den weiteren Gremien zur Entscheidung. Jahressteuergesetze sind im eigentlichen Sinne Bereini-

58 BT-Drs. 16/6518.

59 Vgl. im Einzelnen mit weiteren Hinweisen, Aktueller Begriff der Wissenschaftlichen Dienste des Deutschen Bundestags, Nr. 02/08 vom 4. Februar 2008, http://www.bundestag.de/wissen/analysen/2008/Neuregelung_des_Versicherungsaufsichtsgesetzes.pdf (Abruf am 8. März 2009).

60 Siehe hierzu Volker Müller, Mehr Spielraum gewünscht, in: Das Parlament vom 29. Oktober 2007. 
gungsgesetze der Steuergesetze, die jährlich verabschiedet werden und ihren Ursprung in Entscheidungen oberster Gerichte und in Änderungen der Verwaltungspraxis haben. Daher durchliefen die Jahressteuergesetze in der Vergangenheit auch oftmals unbemerkt für die breite Öffentlichkeit das Gesetzgebungsverfahren. Zunehmend finden sich darin aber politisch heikle Punkte, zum Beispiel die Dienstwagenbesteuerung oder die Besteuerung von Ehegatten. Daher werden die Inhalte der Jahressteuergesetze inzwischen nicht mehr in Fachkreisen allein diskutiert, sondern die von den einzelnen Maßnahmen Betroffenen äußern sich über die Medien und nehmen direkt mit den zuständigen Politikern Kontakt auf.

Die Koalitionsfraktionen hatten allein zum Jahressteuergesetz 2008 im Finanzausschuss 47 Änderungsanträge vorgelegt, die von der FDP komplett abgelehnt wurden, während die Linksfraktion den allermeisten zustimmte und Bündnis 90/Die Grünen sich überwiegend enthielten. Ein zentraler Gegenstand, die Einführung eines optionalen Anteilsverfahrens für berufstätige Ehepaare als Alternative zur Wahl des statischen Lohnsteuerabzug der Steuerklassen III und V, wurde komplett aus dem Regierungsentwurf herausgenommen. ${ }^{61}$ Breiten Raum in der Diskussion zwischen den Koalitionspartnern sowie im Finanzausschuss nahmen Änderungen an der Neuformulierung von $\$ 42$ der Abgabenordnung ein, der den Missbrauch steuerlicher Gestaltungsmöglichkeiten zum Gegenstand hat. In der Anhörung des Ausschusses zum Jahressteuergesetz war unter anderem kritisiert worden, dass mit dem unbestimmten Rechtsbegriff einer „ungewöhnlichen Gestaltung“ operiert werde. In der jetzt geänderten Fassung heißt es, dass ein Missbrauch dann vorliegt, wenn eine „unangemessene" rechtliche Gestaltung gewählt wird, die beim Steuerpflichtigen zu einem „gesetzlich nicht vorgesehenen Steuervorteil“ führt. Ein Missbrauch soll nur dann nicht gegeben sein, wenn der Steuerzahler für seine Gestaltung „außersteuerliche Gründe“ nachweisen kann, die „nach dem Gesamtbild der Verhältnisse beachtlich sind“. Diese Änderungen konnten erst auf der Ebene der finanzpolitischen Sprecher nach langen Diskussionen erzielt werden. Eine weitere Änderung, die erst von den finanzpolitischen Sprechern und auf Ebene der Stellvertretenden Fraktionsvorsitzenden durchgesetzt werden konnte, betrifft eine Maßnahme aus der Unternehmenssteuerreform, die am 1. Januar 2008 in Kraft trat. Damals war die gewerbesteuerliche Hinzurechnung von Finanzierungsanteilen neu geregelt und der Finanzierungsanteil aus Miet- und Pachtzinsen für die Benutzung von unbeweglichen Wirtschaftsgütern des Anlagevermögens auf 75 Prozent festgelegt worden. Die große Koalition verringerte diesen Anteil auf 65 Prozent nach ausgiebigen Beratungen in der Runde der finanzpolitischen Sprecher und der Stellvertretenden Fraktionsvorsitzenden. Der Finanzausschuss nahm schließlich im November 2007 den Entwurf der Bundesregierung für ein Jahressteuergesetz 2008 mit der Mehrheit der Koalitionsfraktionen in geänderter Fassung an. Alle drei Oppositionsfraktionen stimmten gegen das Regelwerk.

\subsection{Unternehmenssteuerreform 2008}

Die Reform der Unternehmensbesteuerung ist eines der wichtigsten Vorhaben der großen Koalition in dieser Wahlperiode. Im Koalitionsvertrag vom 11. November 2005 heißt es: „Deutschland muss auch in Zukunft im internationalen Steuerwettbewerb bestehen können. Deshalb werden in dieser Legislaturperiode zum 1. Januar 2008 das Unternehmens-

61 In überarbeiteter Fassung soll dieser Punkt zu einem späteren Zeitpunkt beraten werden. 
steuerrecht grundlegend fortentwickelt und international wettbewerbsfähige Steuersätze realisiert. Diese Reform umfasst neben den Körperschaften auch die Personenunternehmen, da deutsche Unternehmen zu mehr als 80 Prozent in dieser Rechtsform organisiert sind. (...) Dabei werden uns insbesondere folgende Zielsetzungen leiten: Verbesserung der internationalen Wettbewerbsfähigkeit und Europa-Tauglichkeit, weitgehende Rechts- und Finanzierungsneutralität, Einschränkung der Gestaltungsmöglichkeiten, Verbesserung der Planungssicherheit für Unternehmen und öffentliche Haushalte sowie eine nachhaltige Sicherung der deutschen Steuerbasis. "Zur Besteuerung von Kapitalerträgen heißt es im Koalitionsvertrag: „In dieser Legislaturperiode werden wir eine Neuregelung der Besteuerung von Kapitalerträgen und privaten Veräußerungsgewinnen realisieren. “

Der Entscheidung des Bundestags für die Unternehmenssteuerreform waren langwierige Vorbereitungen und Verhandlungen vorausgegangen. Mehrere strittige Fragen zwischen den Koalitionspartnern - teilweise aber auch parteiintern - bargen schwierige Problemstellungen in sich, zu verschieden waren die Ausgangspositionen. Schließlich ist das Interesse in der SPD an einer Senkung von Steuersätzen an sich nicht besonders ausgeprägt, da sich hierdurch die Einnahmen des Staates und damit sein Gestaltungsspielraum vermindern. Im April 2006 legte der Sachverständigenrat zur Begutachtung der gesamtwirtschaftlichen Entwicklung seine Expertise zur Unternehmenssteuerreform inklusive Reformvorschlägen vor. ${ }^{62}$ Den Auftrag für das Gutachten hatten das Bundesfinanz- und das Bundeswirtschaftsministerium 2005 erteilt. Wenige Wochen später veröffentlichte auch die unabhängige Stiftung Marktwirtschaft Vorschläge zur Reform der Unternehmensbesteuerung in Deutschland. ${ }^{63}$ Sowohl der Sachverständigenrat als auch die Stiftung Marktwirtschaft rechneten mit erheblichen Mindereinnahmen bei einer Umsetzung ihrer Vorschläge. So sprach der Sachverständigenrat selbst von Steuerausfällen in Höhe von 22 Milliarden Euro, und das Modell der Stiftung Marktwirtschaft hätte Aufkommenseinbußen von rund 16 Milliarden Euro verursacht. Allerdings bezogen beide Institutionen keine Vorgaben für eine Gegenfinanzierung ein, anders als dies bei den Verhandlungen zwischen den Koalitionspartnern zur

62 Beim Modell des Sachverständigenrats unterliegen grundsätzlich sämtliche Kapitaleinkommen einem ermäßigten Steuersatz von 25 Prozent, während es bei den übrigen Erwerbseinkommen beim linear-progressiven Steuertarif bleibt (Duale Einkommensteuer). Als Kapitaleinkommen gilt auch die typisierte Eigenkapitalverzinsung, so dass bei gewinnstarken Personenunternehmen dieser so genannte Normalgewinn (zum Beispiel sechs Prozent) einer geringeren Belastung unterliegt als der restliche Gewinn. Bei Kapitalgesellschaften soll der Gewinn ebenfalls mit insgesamt 25 Prozent belastet werden; aus Vereinfachungsgründen erfolgt die steuerliche Privilegierung des Normalgewinns allerdings auf Ebene der Anteilseigner. Immobilieneinkünfte könnten ebenfalls in die Duale Einkommensteuer einbezogen werden, da auch diese Einkünfte auf dem Einsatz von Kapital beruhen. Da auch Fremdkapitalzinsen regelmäßig mit 25 Prozent belastet werden sollen, eignet sich das Modell des Sachverständigenrats gut für die mögliche Einführung einer Abgeltungssteuer.

63 Die Stiftung Marktwirtschaft strebte eine einheitliche Steuer für alle Unternehmen unabhängig von der Rechtsform an, nach der im Unternehmen verbleibende Gewinne mit 22 Prozent besteuert werden. Hinzu kommen - als teilweiser Ersatz für die Gewerbesteuer - weitere acht Prozentpunkte als kommunale Unternehmenssteuer. Dies führt zu einer Gesamtbelastung von insgesamt 30 Prozent. Kommt es zu Ausschüttungen oder Entnahmen, werden diese in Fortentwicklung des Halbeinkünfteverfahrens entsprechend nachbelastet. Flankiert wird die Unternehmenssteuer durch eine Kleinunternehmerregelung, die es insbesondere Einzelunternehmern ermöglicht, in der Einkommensteuer zu verbleiben. Diese Regelung soll greifen, wenn der Gewinn eines Personenunternehmens nachhaltig 120.000 Euro nicht überschreitet. 
Bedingung gemacht wurde. Im Juni 2006 kam eine Arbeitsgruppe „Reform der Unternehmenssteuerreform in Deutschland" von Union und SPD zu Beratungen zusammen, die das Bundesfinanzministerium zuvor vorangetrieben hatte. ${ }^{64}$ Die Arbeitsgruppe umfasste jeweils sechs Vertreter von Union und SPD. Für die Union waren dies Bundeswirtschaftsminister Michael Glos, der hessische Ministerpräsident Roland Koch, Kanzleramtschef Thomas de Maizière, der bayerische Finanzminister Kurt Falthauser, der Stellvertretende Vorsitzende der Unionsfraktion Michael Meister und ihr finanzpolitischer Sprecher Otto Bernhardt. Für die SPD nahmen neben Bundesfinanzminister Peer Steinbrück Finanzstaatssekretär Axel Nawrath, der SPD-Fraktionsvize Joachim Poß, der finanzpolitische Sprecher der Fraktion Jörg-Otto Spiller, die Parlamentarische Staatssekretärin im Bundesfinanzministerium Barbara Hendricks sowie der rheinland-pfälzische Finanzminister Ingolf Deubel teil.

Die Arbeiten der Gruppe wurden von einer technischen AG vorbereitet, in der Vertreter auf Arbeitsebene aus den Koalitionsfraktionen, dem Bundesfinanzministerium und den Landesfinanzministerien vertreten waren. Am 3. Juli 2006 stimmte der Koalitionsausschuss den Eckpunkten für die Unternehmenssteuerreform zu, am 12. Juli 2006 folgte das positive Votum des Kabinetts. ${ }^{65}$ Allerdings ließ die Einigung zunächst viele Detailfragen offen. Daher schlossen sich weitere regelmäßige Verhandlungen der AG an. Diese verliefen grundsätzlich nach dem Schema, dass sich CDU/CSU und SPD zunächst getrennt trafen. Die anschließenden gemeinsamen Sitzungen wurden wiederholt für Abstimmungen von Koch und Steinbrück unterbrochen. Die Ergebnisse wurden stets in die CDU/CSU-Bundestagsfraktion rückgekoppelt und mit dieser abgestimmt. Zunächst wurden der Fraktionsvorsitzende und die federführende Arbeitsgruppe Finanzen sowie die mitberatende AG Wirtschaft und der Parlamentskreis Mittelstand informiert; anschließend wurde die Gesamtfraktion in die Willensbildung mit einbezogen.

Die Arbeitsgruppe verständigte sich schließlich am 2. November 2006 auf ein detailliertes Reformkonzept, das als Grundlage für die Ausarbeitung des späteren Gesetzentwurfs diente. Am 6. November 2006 stimmte der Koalitionsausschuss den gefundenen Kompromissen zu. Die AG unter Leitung von Peer Steinbrück und Roland Koch bereitete in der Folgezeit den Gesetzentwurf mit vor. Sie billigte die letzten strittigen Details und das Gesamtpaket Anfang Februar 2007. Im März 2007 brachte das Kabinett mit seiner Entschei-

64 Dass eine Arbeitsgruppe von Union und SPD die Eckpunkte der Unternehmenssteuerreform vorbereiten sollte, war das Ergebnis eines Treffens von Bundeskanzlerin Angela Merkel (CDU) und Bundesfinanzminister Peer Steinbrück (SPD) Mitte Juni 2006, nachdem letzterer ein Papier seines Hauses zu einer Reform der Unternehmensbesteuerung öffentlich gemacht hatte.

65 Das Bundeskabinett verständigte sich auf folgende Eckpunkte für eine Reform: 1. Die bisherige Körperschaftsteuer und Gewerbesteuer werden durch eine föderale und eine kommunale Unternehmenssteuer ersetzt. Beide Steuern bekommen darüber hinaus eine gemeinsame, einheitliche Bemessungsgrundlage. 2. Die nominale steuerliche Gesamtbelastung der Körperschaften wird von heute etwa 38,65 Prozent auf knapp unter 30 Prozent gesenkt. Neben den Körperschaften werden auch die der Einkommenssteuer unterliegenden Personenunternehmen von der Reform profitieren. Es wird geprüft, ob dies am besten durch eine Investitionsrücklage oder durch eine generelle Begünstigung des im Unternehmen einbehaltenen Gewinns geschehen kann. 3. Geprüft werden auch Maßnahmen gegen den Verlust von Steuersubstrat durch Fremdfinanzierung und zur Verstetigung der kommunalen Finanzen. 4. Es soll ferner eine Abgeltungssteuer auf Kapitalerträge eingeführt werden. 5. Bei der Erbschaftsteuer soll die Unternehmensnachfolge erleichtert werden, indem bei Fortführung des Unternehmens eine steuerliche Privilegierung gelten soll. 
dung den Gesetzentwurf formal auf den Weg. ${ }^{66}$ In der Anhörung zur eigentlichen Reform der Unternehmensbesteuerung am 25. April 2007 nahmen 70 Sachverständige sieben Stunden lang gegenüber den Abgeordneten des Bundestags Stellung, und in der dreistündigen Anhörung des Finanzausschusses zur Einführung der Abgeltungssteuer am 7. Mai 2007 waren 30 Sachverständige anwesend. In den sich anschließenden Gesprächen auf Ebene der Berichterstatter, in der Spiller-Bernhardt-Runde und der Meister-Poß-Runde wurden zahlreiche Änderungen am Gesetzentwurf beschlossen. ${ }^{67}$

Die Koalitionsfraktionen hatten dem Finanzausschuss schließlich 41 Änderungsanträge zum ursprünglichen Gesetzentwurf vorgelegt, denen neben CDU/CSU und SPD teilweise auch die Linksfraktion zustimmte. Die FDP enthielt sich bei der Abstimmung über die Änderungen wie auch die Bündnisgrünen, die allerdings in einigen Fällen mit Nein votierten. Nach viereinhalbstündiger Beratung nahm der Finanzausschuss am 23. Mai 2007 die gleichlautenden Gesetzentwürfe der Koalitionsfraktionen ${ }^{68}$ und der Bundesregierung ${ }^{69}$ für das Unternehmenssteuerreformgesetz 2008 in geänderter Fassung an. Die Oppositionsfraktionen stimmten gegen die Reform. Am 25. Mai 2007 verabschiedete der Bundestag das Gesetz über die Unternehmenssteuerreform mit der Mehrheit der großen Koalition. Der Bundesrat stimmte abschließend am 6. Juli $2007 \mathrm{zu}$.

Die Ergebnisse der Verhandlungen zur Unternehmenssteuerreform in verschiedenen Gruppen zu allen Stadien des Gesetzgebungsverfahrens lassen sich wie folgt zusammenfassen: Im Mittelpunkt stand die Absenkung der Steuerbelastung für alle Unternehmen auf unter 30 Prozent und damit auf ein international wettbewerbsfähiges Niveau. Isoliert betrachtet hätten diese Maßnahmen zu Steuermindereinnahmen in der Größenordnung von 30 Milliarden Euro geführt. Zwischen den Koalitionsfraktionen war aber vereinbart worden, die Steuerausfälle durch die Unternehmenssteuerreform auf fünf Milliarden Euro zu

66 BT-Drs. 16/4841.

67 Eine wesentliche Änderung, die zu jährlichen Steuermindereinnahmen von 400 Millionen Euro führt, ist die Aufnahme der Abschreibungen in die Ausgangsgröße für den Abzug von Zinsaufwendungen im Zuge der geplanten „Zinsschranke“. Ziel der Zinsschranke ist es, durch eine Beschränkung des Betriebskostenabzugs von Zinsen steuerschädliche Gestaltungen von Konzernen zu verhindern, die dazu dienen, in Deutschland entstandenen Gewinn ins Ausland zu verschieben. Eine weitere Änderung, die mit Mindereinnahmen von 160 Millionen Euro verbunden ist, betrifft die Anhebung des möglichen Sofortabzugs von Anschaffungs- oder Herstellungskosten bei so genannten geringwertigen Wirtschaftsgütern von 100 Euro auf 150 Euro. In den Gesetzentwürfen war noch vorgesehen, die jetzige Sofortabzugsgrenze von 410 Euro auf 100 Euro abzusenken. Der geplante Investitionsabzugsbetrag für mittelständische Unternehmen (\$7g Einkommensteuergesetz), der die jetzige Ansparrücklage ablöst, soll von Unternehmen mit einem Betriebsvermögen von bis zu 235.000 Euro in Anspruch genommen werden können. Im Gesetzentwurf lag das höchstzulässige Betriebsvermögen noch bei 210.000 Euro. Ferner wurden geschäftsübliche Skonti und vergleichbare wirtschaftliche Vorteile aus der Hinzurechnungsregelung zur Ermittlung der Gewerbesteuerschuld herausgenommen. Die Mindereinnahmen sollen vor allem mit einer Beschränkung der Verlustverrechnung bei Veräußerungsgeschäften mit Aktien im Zusammenhang mit der Abgeltungsteuer gegenfinanziert werden. Verluste aus privaten Veräußerungsgeschäften mit Aktien sollen nun lediglich mit Gewinnen aus solchen Veräußerungsgeschäften verrechnet werden können. Nach dem Gesetzentwurf waren diese Verluste auch noch mit Einkünften aus anderem Kapitalvermögen, etwa Zins- oder Dividendeneinkünften, verrechenbar. Die Koalition erhofft sich davon Mehreinnahmen von 450 Millionen Euro.

68 BT-Drs. 16/4841.

69 BT-Drs. 16/5377. 
begrenzen, so dass gleichzeitig zur Gegenfinanzierung Regelungen getroffen werden mussten, die die Besteuerung in Deutschland erwirtschafteter Gewinne sichern und die Sanierung der öffentlichen Haushalte nicht in Frage stellen würden. ${ }^{70}$ Gerade die im Zusammenhang mit der Reduzierung der Mindereinnahmen in Höhe von insgesamt fünf Milliarden Euro verbundenen belastenden Maßnahmen für die Unternehmen waren zwischen den Koalitionsfraktionen heftig umstritten. Mit der Unternehmenssteuerreform verbunden ist die Einführung einer Abgeltungssteuer mit einem Steuersatz von 25 Prozent auf Kapitalerträge und private Veräußerungsgewinne. Damit sollen den Anlegern attraktive ertragsteuerliche Rahmenbedingungen angeboten werden, und Kontrollverfahren können entfallen.

Dieses Gesetzgebungsverfahren zeigt, dass lange um Inhalte und Formulierungen zwischen den Koalitionspartnern gerungen werden musste - sowohl in der Arbeitsgruppe vor Erstellung des Referentenentwurfs als auch im parlamentarischen Verfahren zwischen den Berichterstattern, Arbeitsgruppensprechern und Stellvertretenden Fraktionsvorsitzenden. Die Unternehmenssteuerreform ist ein Paradebeispiel für das Wirken der verschiedenen formellen und informellen Institutionen und Entscheidungsverfahren der großen Koalition auf Politikfeldern, wo die Koalitionspartner von ihrer Grundausrichtung weit auseinander liegen. Gleichzeitig wird deutlich, dass in diesen Ausgangskonstellationen allein Kompromisse erzielt werden können, die für beide Seite nicht die Ideallösung darstellen.

\section{Fazit: Große Koalitionen als Zweckbündnis mit besonderen Spielregeln}

Zwischen den großen Koalitionen von 1966 bis 1969 und seit 2005 zeigen sich einige Parallelitäten $^{71}$, auch wenn die Ausgangslagen und das politische Umfeld sehr unterschiedlich sind. „Im Gegensatz zur Großen Koalition seit 2005, bei der die personalen Strukturen an der Spitze offenkundig sehr funktional geordnet sind und jeweils die politischen Spitzen

70 Im Einzelnen: Maßnahmen zur Stärkung von Wachstum und Beschäftigung: (1) Verringerung des Körperschaftsteuersatzes von 25 Prozent auf 15 Prozent; (2) Senkung der Gewerbesteuermesszahl von max. fünf Prozent auf einheitlich 3,5 Prozent; (3) Erhöhung des Anrechnungsfaktors von 1,8 auf 3,8 zur Anrechnung der Gewerbesteuer auf die Einkommensteuer; (4) Einführung einer Thesaurierungsbegünstigung von 28,25 Prozent (zuzüglich Soli) für im Unternehmen belassene laufende Gewinne; (5) Ausbau der Ansparrücklage zu einem Investitionsabzugsbetrag ( $\$ 7 \mathrm{~g} \mathrm{EStG);}$ (6) ab 1. Januar 2009 Einführung einer Abgeltungssteuer von 25 Prozent (zuzüglich Soli) auf Einkünfte aus Kapitalerträgen und Veräußerungsgewinnen mit Veranlagungswahlrecht. Maßnahmen zur Sicherung des Steuersubstrats und zur Finanzierung: (1) Wegfall Abzugsfähigkeit der Gewerbesteuer als Betriebsausgabe; (2) Wegfall Staffeltarif von ein Prozent bis fünf Prozent bei Personenunternehmen; (3) Ersatz der 50-prozentigen Hinzurechnung von Dauerschuldzinsen durch eine 25-prozentige Hinzurechnung aller Zinsen und aller Finanzierungsanteile von Mieten, Pachten, Leasingraten und Lizenzgebühren (Freibetrag 100.000 Euro); (4) Einführung einer Zinsschranke (Begrenzung des Betriebsausgabenabzugs für Zinsaufwendungen) mit Ausnahmen: Freigrenze von einer Million Euro, Beschränkung auf konzernzugehörige Betriebe und gegebenenfalls Eingreifen der Escape-Klausel, also wenn die Eigenkapitalquote des Betriebs die Eigenkapitalquote des Konzerns nicht unterschreitet; (5) gesetzliche Ausgestaltung der Verrechnungspreisbildung mit entsprechenden Regelungen für Funktionsverlagerungen; (6) Einschränkungen der Gestaltungen bei der Wertpapierleihe; (7) Neuregelung der Verlustabzugsbegrenzung beim so genannten Mantelkauf; (8) Abschaffung der degressiven AfA; (9) Neuregelung der Sofortabschreibung von geringwertigen Wirtschaftsgütern (GWG).

71 Einen ersten Vergleich zieht Joachim Samuel Eichhorn, a.a.O. (Fn. 9), S. 244 f. 
untereinander die zentralen Kommunikationen austragen, prägte die informellen Führungszirkel der Großen Koalition von 1966-1969 noch ein stark männerbündisches Moment. "72 In der Rückschau scheint nicht eindeutig zu sein, wem die Hauptführerschaft in der großen Koalition damals zuzuordnen ist (Bundeskanzler, Fraktionsführer oder Parteivorsitzende). ${ }^{73}$ Der Fraktionsvorsitzende der SPD Schmidt betonte bereits 1966, man dürfe die grundgesetzlich vorgegebene Richtlinienkompetenz des Bundeskanzlers nicht überschätzen. Diese Einschätzung teilte offenbar auch Bundeskanzler Kiesinger ${ }^{74}$, und daran dürfte sich auch heute nichts Wesentliches geändert haben.

Vorstufe für das Zusammenwirken im Kabinett ist die Koordination der Regierungsparteien. ${ }^{75}$ In der großen Koalition seit 2005 ist diese institutionalisiert worden. Die Minister treffen jeweils getrennt nach ihrer Fraktionszugehörigkeit vor den Kabinettssitzungen zusammen. Dabei sind die Fraktionsvorsitzenden eingebunden. Die Fraktionen selbst spielen in einer großen Koalition eine ungemein große Rolle, damals wie heute. ${ }^{76}$ Unter anderem werden Gesetzentwürfe nicht nur parallel durch die Fraktionen in den Bundestag eingebracht, um das Verfahren zu beschleunigen. Vielmehr dient dies auch einer stärkeren Bindung an die Gesetze selbst und fördert das einvernehmliche Handeln. ${ }^{77}$ Möglich ist ebenso, dass die Koalitionsfraktionen Entwürfe der Bundesregierung nicht nur in Einzelfragen, sondern auf eigene Initiative, teilweise gegen den ausdrücklichen Willen des federführenden Ministeriums, auch in Kernpunkten abändern oder ergänzen. ${ }^{78}$

Damals wie heute ist eine gewisse Nervosität bei den Koalitionsfraktionen zu vernehmen, denn offenbar verringern große Koalitionen den Parteienwettbewerb. Zumindest ein, wenn nicht sogar alle Partner „leiden“ unter den notwendigen Kompromissen in Mei-

72 Peter März, a.a.O. (Fn. 8), Kapitel 7, S. 6.

73 Joachim Samuel Eichhorn, a.a.O. (Fn. 9), S. 233 und dort insbesondere Fußnote 10 mit weiteren Hinweisen.

74 Ebenda, S. $234 \mathrm{f}$.

75 Zumindest 1966 bis 1969 schienen die Sozialdemokraten es hier leichter zu haben als die Union. Die neun SPD-Kabinettsmitglieder trafen sich regelmäßig zu Aussprachen und „wurden darauf präpariert, im Kabinett als Block abzustimmen“. Christ und Welt vom 12. Januar 1968, Geschlossene Gesellschaft. Kreßbronner Kreis - Legende oder Wirklichkeit? Allerdings fand auch in der CDU/CSU eine vorherige Zusammenkunft der Unions-Minister statt, und zwar dienstagabends vor der Kabinettssitzung. Vgl. Joachim Samuel Eichhorn, a.a.O. (Fn. 9), S. 240.

76 Nach Klaus Schönhoven, a.a.O. (Fn. 31), S. 177 hatten sich die beiden Fraktionsvorsitzenden Schmidt und Barzel im Herbst 1967 darauf verständigt, in beiden Fraktionen nur solche Beschlüsse zu verabschieden, die jeder Fraktion bei Prestigefragen noch Spielraum ließen.

77 Im Koalitionsvertrag unter Punkt II. Kooperation der Fraktionen heißt es zum einvernehmlichen Handeln der Koalitionsfraktionen: „Im Bundestag und in allen von ihm beschickten Gremien stimmen die Koalitionsfraktionen einheitlich ab. Das gilt auch für Fragen, die nicht Gegenstand der vereinbarten Politik sind. Wechselnde Mehrheiten sind ausgeschlossen. Über das Verfahren und die Arbeit im Parlament wird Einvernehmen zwischen den Koalitionsfraktionen hergestellt. Anträge, Gesetzesinitiativen und Anfragen auf Fraktionsebene werden gemeinsam oder, im Ausnahmefall, im gegenseitigen Einvernehmen eingebracht. Die Koalitionsfraktionen werden darüber eine Vereinbarung treffen."

78 Beispielsweise haben die Koalitionsfraktionen im Risikobegrenzungsgesetz (BT-Drs. 16/9821) den dort enthaltenen Prüfauftrag zur Fragestellung der Kreditverkäufe mit eigenen Formulierungen ausgefüllt. Im Jahressteuergesetz 2009 erfolgte allein auf Initiative der Koalitionsfraktionen im Gesetzgebungsverfahren eine Formulierung für Leasing- und Factoringunternehmen zur Einbeziehung in das gewerbesteuerrechtliche Bankenprivileg, da ansonsten in Folge der Unternehmenssteuerreform es zu nicht sachgerechten Belastungen kommt. 
nungsumfragen. So war es gegen Ende der ersten großen Koalition, so ist es heute wieder. Als Bundeskanzler Kiesinger in seiner Rede auf dem Bundesparteitag der CDU 1968 klar machte, dass auch nach 1969 eine Koalition mit der SPD wahrscheinlich sein könnte, erntete er Widerspruch und Empörung. ${ }^{79}$ In der Bevölkerung aber nahm der Wunsch nach Beibehaltung der großen Koalition zu. Der Wunsch nach Harmonie überwog hier. ${ }^{80}$ Derzeit ist offen, ob die Wähler tatsächlich eine weitere Wahlperiode von einer großen Koalition regiert werden wollen. ${ }^{81}$ Viele Meinungsumfragen sprechen derzeit eher dagegen. Im Zögern der SPD, sich mit der Union auf ein neues Wahlrecht zu einigen, sah man 1969 einen Schwenk hin zur FDP. Auf sie setzten die Sozialdemokraten auch bei der Wahl des Bundespräsidenten, für die sie als Symbol der Eigenständigkeit einen eigenen Kandidaten benannt hatten. Am Ende unterlag der Unionskandidat Gerhard Schröder dem populären Gustav Heinemann, so dass das höchste Staatsamt der Bundesrepublik zum ersten Mal von einem Sozialdemokraten bekleidet wurde. Ob sich in diesem Punkt die Geschichte wiederholen wird, wird sich zeigen. Nach anfänglich anderen Bekundungen hat die SPD ebenfalls für die Wahl des Bundespräsidenten im Jahre 2009 mit Gesine Schwan eine Gegenkandidatin zu Horst Köhler aufgestellt.

1966 handelte es sich zwar ebenso wenig um eine „Liebesheirat“ wie 2005, allerdings war die erste große Koalition lange vorbereitet worden. Große Koalitionen sind und bleiben ein Zweckbündnis mit besonderen Spielregeln. Heute wie vor vierzig Jahren ist klar, dass ein Scheitern den Unmut in der Bevölkerung über das demokratische System stärken und Parteien am Rande des politischen Spektrums weiteren Zulauf bescheren würde. Daher sind Kompromisse letztlich das einzige Mittel, um Ergebnisse herbeizuführen, mögen die Gegensätze auch noch so tiefgreifend sein. Ein Dissensmanagement darf die Tagesordnung nicht bestimmen. ${ }^{82}$ Daher haben sich auf allen Ebenen, sowohl innerhalb der beiden Fraktionen als auch zwischen ihnen, informelle Gremien zur Willensbildung zusammengefunden, die mehr oder minder institutionalisiert tagen, wie am durchaus verallgemeinerungsfähigen Beispiel der Finanzpolitik gezeigt werden konnte. Große Koalitionen sind „zum Erfolg verurteilt“, umso wichtiger ist daher die Abstimmung und breite Willensbildung.

Wenn in Zukunft fünf Fraktionen die Regel im Bundestag und in den Landesparlamenten sein werden, dürfte die Koalitionsbildung umso schwieriger werden. Die FDP musste schon durch die Erfolge der Grünen lernen, dass sie sich nicht mehr auf ihre lange in der alten Bundesrepublik gehegte Rolle des „Züngleins an der Wage“ für einen Regierungs-

79 Reinhard Schmoeckel/ Bruno Kaiser, a.a.O. (Fn. 5), S. 81.

80 Unter den Anhängern der Union neigten 58 Prozent zum Beibehalten der großen Koalition, während im Sommer 1969 bei der SPD 41 Prozent dieser Ansicht waren, vgl. Hans Heigert, Sentimentale Sehnsucht nach Harmonie. Warum immer mehr Leute sagen, Große Koalition solle bleiben, in: SZ vom 5. September 1969.

81 Inzwischen kommt aber auch von Regierungsmitgliedern der Hinweis, dass allein eine weitere Wahlperiode mit einer großen Koalition zuverlässig die Politik der nächsten Jahre bestimmen könne, so etwa Peer Steinbrück in einem Interview mit der Bild-Zeitung vom 14. Juli 2008. Andere Stimmen kritisieren, dass der „Konsens der Mitte“ gerade Parteien am Rand wie die Linke beflügelt, vgl. Wolfgang Münchau, Die Mär von der Verantwortung, in: Financial Times Deutschland vom 23. Juli 2008; siehe auch Karlheinz Niclauß, a.a.O. (Fn. 57), S. 6 mit weiteren Hinweisen.

82 Vgl. Karl-Rudolf Korte, a.a.O. (Fn. 42), S. 90. Er hält die derzeitige große Koalition aber für ein Bündnis geprägt vom Dissensmanagement. 
wechsel durch eine Koalition aus zwei Fraktionen zurückziehen kann. Mit dem Erstarken der Linken auch in Westdeutschland ist eine fünfte Kraft in den Parlamenten vorhanden, die auf Bundesebene und in den westdeutschen Landesparlamenten Regierungsverantwortung noch vor sich haben könnte, die sie in den neuen Bundesländern und Berlin bereits wahrnimmt. Diese Konstellation wird nach menschlichem Ermessen dazu führen, dass absolute Mehrheiten von CDU, CSU oder SPD weitgehend der Vergangenheit angehören beziehungsweise eine Ausnahme bilden werden. Die große Koalition auf Bundesebene seit 2005 und ihre spezifischen formellen wie informellen Willensbildungsprozesse könnten daher ein Leitbild für ähnliche Konstellationen auf Landesebene und auch in Zukunft im Bund sein. 